\title{
Topological classifier for detecting the emergence of epileptic seizures
}

\author{
Marco Piangerelli ${ }^{1 *}$ (D, Matteo Rucco ${ }^{2}$, Luca Tesei ${ }^{1}$ and Emanuela Merelli ${ }^{1}$
}

\begin{abstract}
Objective: An innovative method based on topological data analysis is introduced for classifying EEG recordings of patients affected by epilepsy. We construct a topological space from a collection of EEGs signals using Persistent Homology; then, we analyse the space by Persistent entropy, a global topological feature, in order to classify healthy and epileptic signals.

Results: The performance of the resulting one-feature-based linear topological classifier is tested by analysing the Physionet dataset. The quality of classification is evaluated in terms of the Area Under Curve (AUC) of the receiver operating characteristic curve. It is shown that the linear topological classifier has an AUC equal to 97.2\% while the performance of a classifier based on Sample Entropy has an AUC equal to 62.0\%.
\end{abstract}

Keywords: Complex systems, Brain, Epilepsy, Topological data analysis, Persistent entropy, Time series

\section{Introduction}

Epilepsy is a chronic brain disorder characterised by recurrent seizures of several entity with different manifestations. They are caused by sudden excessive electrical discharges in a group of neurons [1] and they are defined as a spontaneous hyper-synchronous activity of clusters of neurons [2].

Human brain can be considered as a complex selfadaptive system composed of billions of non-identical neurons, entangled in loops of non-linear interactions, determining the brain behaviours [3]. Epilepsy is just an example of such behaviours: identifying the onset of a neural hyper-synchronisation is similar to discovering patterns of information expressed by a network of interactions in the space of neurons.

The electroencephalogram (EEG) is the standard technique used to record the electrical activity of the brain. The direct observation of EEG signals helps neurologists in diagnosing epilepsy while automatic methods for this task are still not used even if, in the last decades, several

\footnotetext{
${ }^{*}$ Correspondence: marco.piangerelli@unicam.it

${ }^{1}$ Computer Science Division, School of Science and Technology,

University of Camerino, Via Madonna delle Carceri, 9, 62032 Camerino, IT, Italy

Full list of author information is available at the end of the article
}

methods for automatic diagnosis have been proposed in the literature [4-8]. The intrinsic non-linearity and nonstationarity of EEG signals requires methods capable of extracting global information, characterising the processes described by the signals.

Topological data analysis (TDA) is able to extract such information [9-14]; currently, it has been used for the analysis of EEG signals [15] within the TOPDRIM project [16]. The key-concept in TDA is persistent homology: a procedure for counting, through a process called filtration, the higher dimensional persistent holes of topological spaces. Its visualisation can be given as persistent barcodes or as persistent diagrams.

In this paper we describe the realisation of a Persistent Entropy-based classifier to discriminate the epileptic EEG signals from the non-epileptic ones. The proposed method defines an automatic classifier of signals and it is a preliminary step towards the study of an automatic detection of epileptic seizures. Afterwards, we use the Vietoris-Rips filtration for understanding how the regions of the brain are involved in the spreading of epileptic signals.

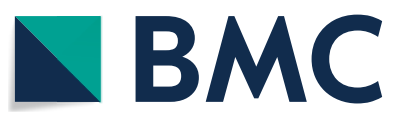

C The Author(s) 2018. This article is distributed under the terms of the Creative Commons Attribution 4.0 International License (http://creativecommons.org/licenses/by/4.0/), which permits unrestricted use, distribution, and reproduction in any medium, provided you give appropriate credit to the original author(s) and the source, provide a link to the Creative Commons license, and indicate if changes were made. The Creative Commons Public Domain Dedication waiver (http://creativecommons.org/ publicdomain/zero/1.0/) applies to the data made available in this article, unless otherwise stated. 


\section{Main text}

\section{Material and methods}

Dataset

The dataset used consists of EEG signals, i.e. multivariate time series (see Fig. 1a), taken from the PhysioNet database [17]. EEGs are performed positioning electrodes at some key points on the patient's head following some schemes: the database we used adopts the international 10-20 system (see Fig. 1c). The EEGs used in this study were collected at the Children's Hospital Boston and they consist of recordings from pediatric subjects with intractable seizures. Subjects were monitored for several days
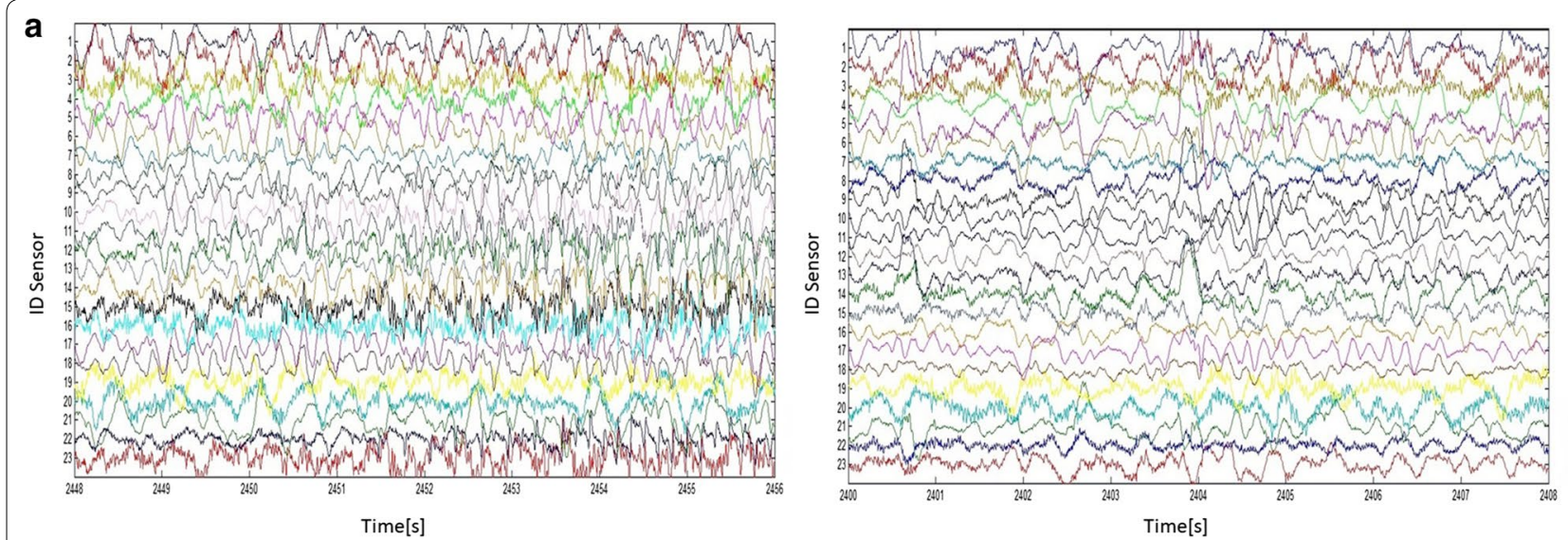

b
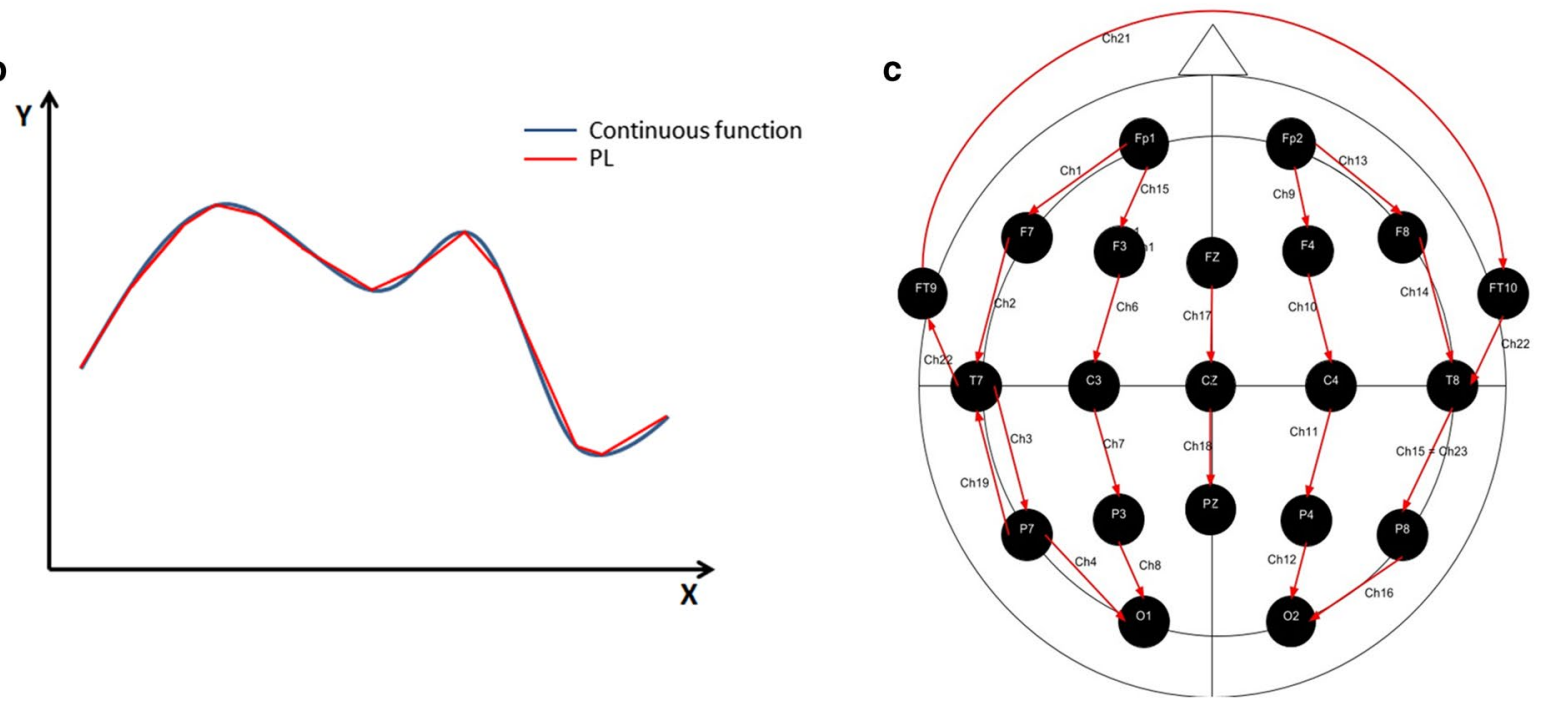
following the withdrawal of anti-seizure medication in order to characterise their seizures and assess their candidacy for surgery. We selected 33 recordings with, at least, one epileptic event and 33 without epileptic events. The recordings have the same number of channels (electrodes), 23, with the same length, 921,600 samples with a sampling frequency of $256 \mathrm{~Hz}$.

\section{TDA: a new method for data analysis}

Consider a set of points $G$, i.e. our data, embedded in a $d$-dimensional space $\mathbb{D}^{d}$ and assume that those data were sampled from an unknown $k$-dimensional space $\mathbb{D}^{k}$ with $k \leq d$. Our task is to reconstruct the space $\mathbb{D}^{k}$ from the dataset $G$.

In TDA, $G$ elements are equipped with a notion of proximity that characterises a coordinate-free metric. Those points are converted into topological spaces called simplicial complexes. Simplicial complexes are made up by building blocks called simplices: points are 0 -simplices, line segments are 1-simplices, filled triangles are 2 -simplices, filled tetrahedra are 3-simplices and so on (see Fig. 1d).

A Filtration is a collection of nested simplicial complexes. Building a filtration can be seen as wearing lenses for examining the dataset: different lenses consent to extract different kinds of information from the topological space. In this paper we use Piecewise filtration and Vietoris-Rips filtration. Choosing a filtration is a crucial step: different filtrations give rise to different conversions of the data points $\mathrm{G}$ into simplicial complexes [18-20].

Piecewise filtration Piecewise filtration, recently introduced by Rucco et al. [21], is used for studying signals. The procedure is based on the well known concept of Piecewise Linear function (PL), $P L: \mathbb{R} \rightarrow \mathbb{R}$, shown in Fig. 2a, b.

Vietoris-Rips filtration Vietoris-Rips filtration is used for studying Point Cloud Data (PCD). It creates a sequence of simplices, built on a metric space, used to add topological structure to an otherwise disconnected set of points [22, Chapter III]. Figure 2c, d, e show a graphical representation of this approach.

Persistent homology Persistent homology is the combinatorial counterpart of Homology, an algebraic object that counts the number of $n$-dimensional holes in a topological space, the so-called Betti numbers. The filtration process is necessary for the computation of persistent homology. The set of Betti numbers is composed by $\beta_{0}$, the number of connected components in a generic topological space $K ; \beta_{1}$, the number of holes in $K ; \beta_{2}$, the number of voids in $K$ and so on. Along the filtration, persistent homology calculates $k$-dimensional Betti intervals: a $k-$ dimensional Betti interval $\left[t_{\text {start }}, t_{\text {end }}\right]$ defines the time at which a $k$-dimensional hole appears in the simplicial complex $\left(t_{\text {start }}\right)$, while $t_{\text {end }}$ is the time at which it disappears. The holes that are still present at $t_{\text {end }}=t_{\max }$ correspond to persistent topological features [23]. A graphical representation of those intervals in $K$ is called persistence barcode and it is associated to a filtration. An equivalent representation is a persistence diagram [24]. An additional information returned by the computation of persistent homology is the list of the generators, which are the simplices involved in the holes. Experimentally, the generators play a crucial role for the description of the data under analysis $[25,26]$.

Persistent entropy A new entropy measure called Persistent entropy has been recently introduced for measuring how much the construction of a filtered simplicial complex is "ordered" [27]. Given a topological space $K$ and a set of the filtration parameters $F$, let $B=\left\{\left[x_{i}, y_{i}\right) \mid i \in I\right\}$, where $i$ is a set of indexes, be the persistent barcode associated to the filtration of $K$. The Persistent entropy $H$ of the filtered simplicial complex is calculated as follows:

$$
H=-\sum_{i \in I} p_{i} \log \left(p_{i}\right)
$$

where $p_{i}=\frac{\ell_{i}}{L}, \ell_{i}=y_{i}-x_{i}$, and $L=\sum_{i \in I} \ell_{i}$. In case of a persistent interval $\left[x_{i}, \infty\right)$, an interval $\left[x_{i}, m\right)$ is used, where $m=\max \{F\}+1$. Moreover, to rescale $H$ in the interval $[0,1]$ and to compare the values from different barcodes we use the stability theorem for $H$ and the normalised $H$, denoted by $\mathcal{H}$, and defined as:

$$
\mathcal{H}=\frac{H}{\log \ell_{\max }}
$$

where $\ell_{\max }$ is the maximum interval length in the considered barcode [21].

\section{A new topological classifier for epilepsy}

Given the above theoretical framework, let us define a new methodology for the analysis of EEG signals. It can be divided in three steps:

Step I preprocessing of the input.

Step II computation of $H$ using the Piecewise filtration and derivation of a linear topological classifier (LTC).

Step III identification of regions involved in the spreading of the epileptic signals using VietorisRips filtration. 


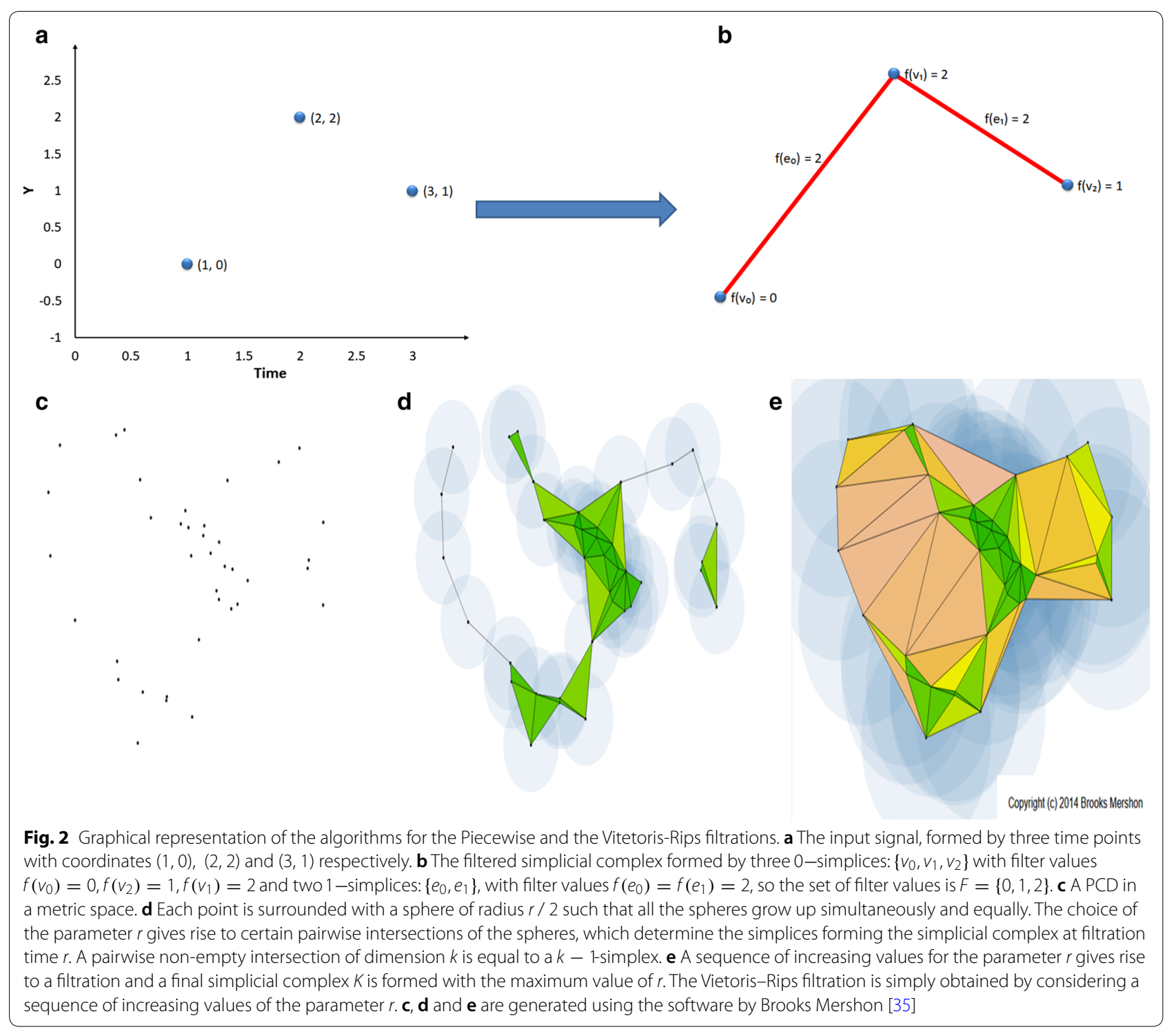

\section{Step I}

Let $j \in\{1,2, \ldots, 66\}$ be the index of the EEG recordings, denoted by $\mathbb{S}^{j}$. Each $\mathbb{S}^{j}$ is composed of 23 one-dimensional signals, $\mathbb{S}^{j}=\left\{S_{1}^{j}, S_{2}^{j}, \ldots, S_{23}^{j}\right\}$, and each $S_{i}^{j} \subset \mathbb{R}^{2}$ is a PL function. The length of each $S_{i}^{j}$ is $N$, the number of samples. For each $S_{i}^{j}$ the preprocessing performs two actions:

1. Filtering the EEG reduces the noise by using a bandpass filter between $1-70 \mathrm{~Hz}$, and removes the power line using a notch filter, between 8 and $52 \mathrm{~Hz}$ [2830].

2. Downsampling the EEG reduces the time needed for the computation of the topological features during the subsequent steps. The worst-case complexity of computing persistent homology using the JavaPlex tool [31] is cubic in the number of simplices. This number is linear with respect to the number of points in case of piecewise complexes. Downsampling should be used if and only if it preserves the main geometrical characteristics of the original signals, that is the shape. In MATLAB we used the command "decimate" [32].

After the preprocessing, the signals were denoted $\tilde{S}_{i}^{j}$.

\section{Step II}

After performing the Piecewise filtration, we computed $\mathcal{H}$ for each $\tilde{\mathbb{S}} j$ thus obtaining a vector of 23 values of $\mathcal{H}$. Then, we calculated the average value of this vector, $\widehat{\mathcal{H}^{j}}$. 

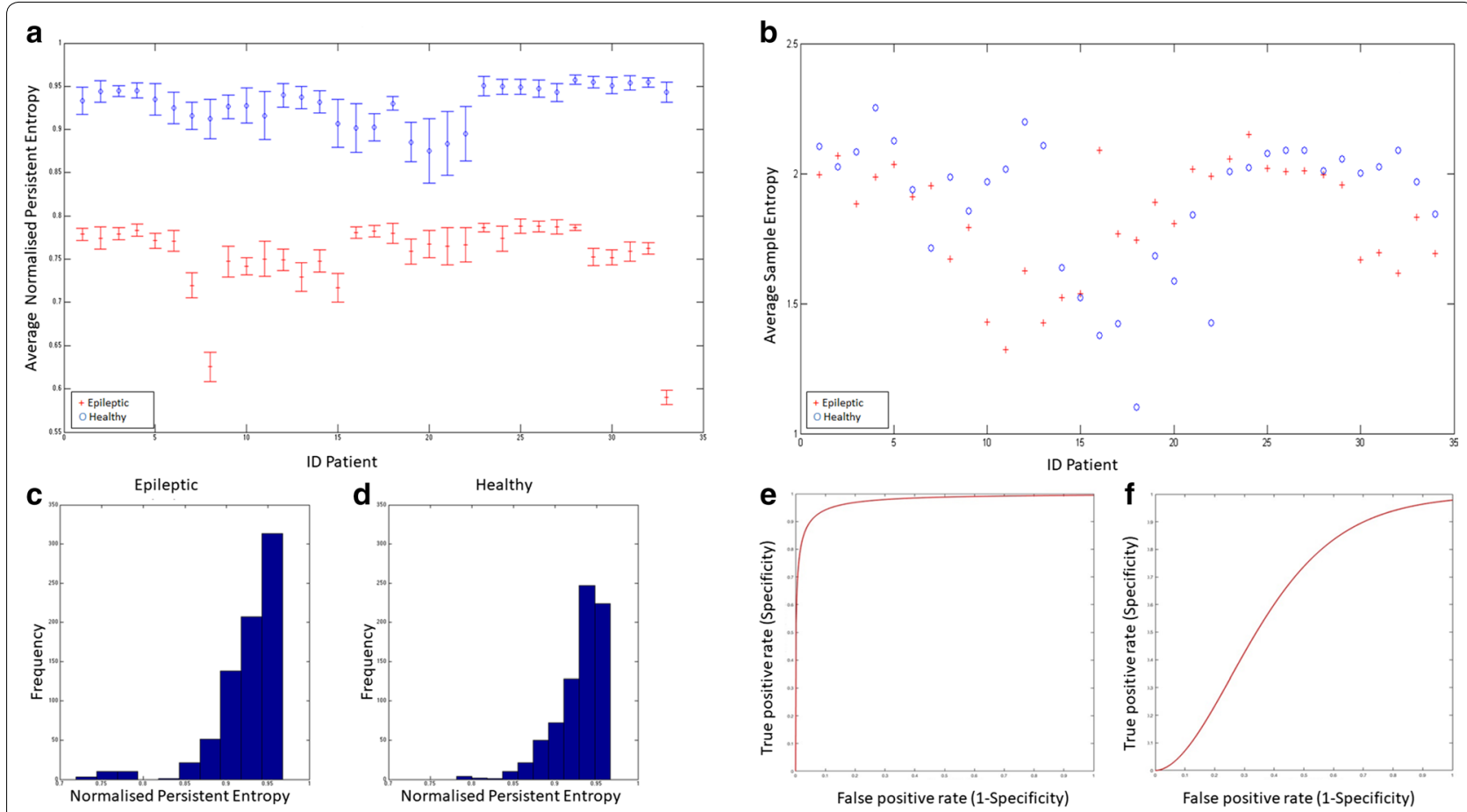

g

Healthy

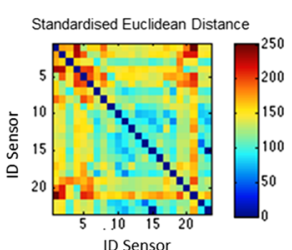

h

Epileptic

i

False positive rate (1-Specificity)

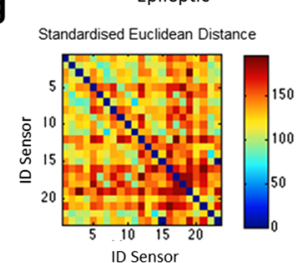

ID Sensor

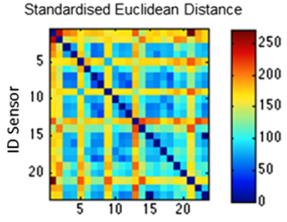

ID Sensor

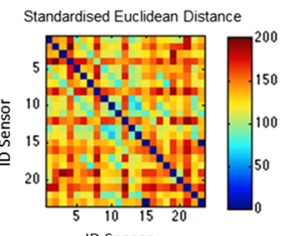

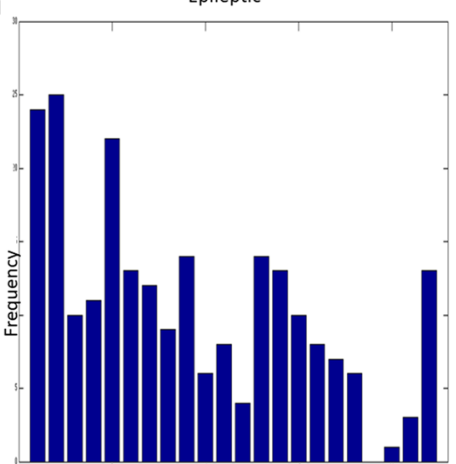

ID Sensor

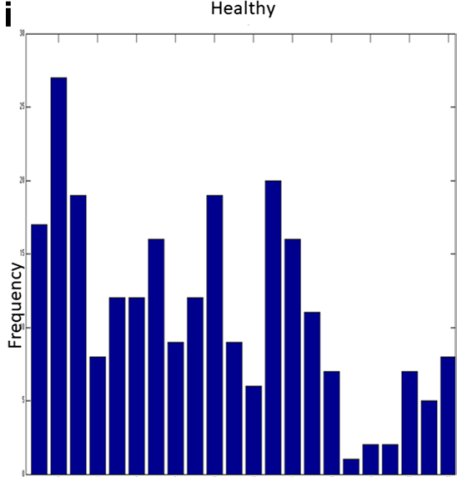

ID Sensor

Fig. 3 a $\widehat{\mathcal{H}^{j}}$ for epileptic (red) and healthy (blue) signals. b Average value of Sample Entropy over the 23 EEG signals of epileptic patients (red) and of healthy patients (blue). c, $\mathbf{d}$ Histogram of the frequencies of $\mathcal{H}^{j}$ values for each channel, for each patient and for the two classes of patients. The elements are sorted into 10 equally spaced bins along the $x$-axis between the minimum and maximum values of $\mathcal{H}^{j}$. e The ROC curve of the $\widehat{\mathcal{H}}$ -based classifier (AUC $=97.2 \%$ ). $\mathbf{f}$ The ROC curve of the Sample Entropy-based classifier (AUC $=62 \%$ ). $\mathbf{g}$ Mosaic Plots representing the distance matrices of the recordings of four patients. The entries of the matrices are the pairwise Standardised Euclidean distances among sensors. The matrices are used in the calculation of Vietoris-Rips filtration. $\mathbf{h}$, i Frequency of the generators, i.e. sensors, belonging to the found i-dimensional holes for 33 epileptic signals (left) and 33 healthy signals (right)

$\widehat{\mathcal{H}^{j}}$ is our 1-dimensional feature able to differentiate signals by looking at their shapes [21].

We repeated the procedure using Sample Entropy, a well-established technique in time series analysis [33, 34], on the same dataset. Finally, we trained an $\mathcal{H}$-based supervised classifier and a Sample Entropy-based supervised LTC. We randomly divided the dataset into a training (70\%) and a testing (30\%) subset. We applied a 10 -fold cross validation.

\section{Step III}

Let us consider our dataset as a PCD: each patient is represented by 23 points in $\mathbb{R}^{N}$. Assuming that the generators of the persistent holes correspond to the sensors on the head of the patient, we applied the Vietoris-Rips 
filtration to determine which particular sensors (thus, which areas of the brain) are more "involved" "significant" concerning the spreading of epileptic seizures. Standardised Euclidean distance among sensors, see Fig. $3 \mathrm{~g}$, is the metric upon which we performed the Vietoris-Rips filtration. This metric is useful when the dataset contains heterogeneous scale variables and it is defined as:

$$
d\left(\tilde{S}, \tilde{S}^{\prime}\right)=\sqrt{\sum_{k=1}^{N}\left(\frac{\left.\tilde{S}\right|_{k}}{s_{k}}-\frac{\left.\tilde{S}^{\prime}\right|_{k}}{s_{k}}\right)^{2}}
$$

where $\left.\tilde{S}\right|_{k}$ stands for the $y$-component in $\left(x_{k}, y_{k}\right)$ of the channel signal $\tilde{S}$ and $s_{k}$ is the sample standard deviation calculated among the $23 y$-components at position $k$ of the signal $\tilde{\mathbb{S}}$ to which $\tilde{S}$ belongs.

\section{Results}

We report the results of the analysis on the signals decimated by a factor 10, which produced 92160 samples per signal $(N=92160)$. We tested our method using the non-downsampling signals and using different values of the decimation factor $(d f=10$ and $d f=100)$. We report the results of the analysis using $d f=10$ (because $H$ did not show significative changes for $d f=100$ ). In Fig. 3c, $d$ the frequency of the values of $\mathcal{H}_{i}^{j}$ is reported. The class of epileptic patients is characterised by a peak of 313 elements in the range [0.942, 0.967] of $\mathcal{H}$ values, with centre value 0.955 . The class of healthy patients is characterised by a peak containing 247 elements with $\mathcal{H}$ values in $[0.930,0.948]$ with centre value 0.939 . A strong separation between the two classes is clearly depicted in Fig. 3a where $\widehat{\mathcal{H}^{j}}$ is plotted. It is evident from the figure that there is a strong separation between the two populations. The Wilcoxon test ( $\mathrm{p}$-value $=1.8346 \mathrm{e}-36$ and confidence interval [1.6942, 1.9675]), used because of the non-normal distribution of classes, confirmed the separation. Sample Entropy failed to separate the two classes, see Fig. 3b.

The receiver operating characteristic (ROC) curves of the two classifiers are shown in Fig. 3e, f. The Area Under Curve (AUC) for the $\widehat{\mathcal{H}}$-based LTC is $97.2 \%$, while the AUC for the Sample Entropy-based classifier is $62 \%$. The $\widehat{\mathcal{H}}$-based classifier ROC curve suggests that the best threshold for the separation of the two classes is $\theta=0.8754$.

For each patient we extracted the values of the Betti numbers: even if there are less epileptic than healthy signals with $\beta_{0}$ ( 3 vs. 12), this difference is not significant ( $\mathrm{p}$-value $=0.6946$, Wilcoxon test). In Fig. $3 \mathrm{~h}$, i the generators of all the found $i$-dimensional holes, were grouped in a frequency histogram. We can recognise that the epileptic patients are characterised by 3 sensors (IDs 1, 2 and 5) while the healthy patients are characterised by sensors with IDs 1, 2, 3, 7, 10, 13 and 14. Those histograms are to be intended quantitatively: sensors involved in epilepsy spread are a few with respect to the ones involved in the normal brain activity.

\section{Limitations}

The results for the classifier are very promising, even if we are aware that the reduced number of samples requires further investigations over the effectiveness of the method. Moreover, the role of generators should be deeply investigated. Nevertheless, we believe the present methodology provides a useful example regarding the use of TDA, especially in time series analysis.

\section{Abbreviations}

AUC: Area Under Curve; EEG: electroencephalogram; H: persistent entropy; PCD: Point Cloud Data; ROC: receiving operative characteristic; TDA: topological data analysis; LTC: linear topological classifier.

\section{Authors' contributions}

MP, MR and EM conceived the project. MP and MR performed the computations. MP wrote the paper. MP, MR, LT and EM contributed in the interpretation of the results. EM and LT critically revised the manuscript. All authors read and approved the final manuscript.

\section{Author details}

${ }^{1}$ Computer Science Division, School of Science and Technology, University of Camerino, Via Madonna delle Carceri, 9, 62032 Camerino, IT, Italy. ${ }^{2}$ ALES S.r.l.-United Technologies Research Center, Via Praga, 5, 38121 Trento, IT, Italy.

\section{Acknowledgements}

The authors thank Giovanna Viticchi, and Riccardo Ricciuti, from AOU - Ospedali Riuniti di Ancona (Italy) for valuable discussions on medical aspects about epilepsy and Alessandra Renieri regarding algebraic issues.

\section{Competing interests}

The authors declare that the research was conducted in the absence of any commercial or financial relationships that could be construed as a potential competing interests.

\section{Availability of data and materials}

The datasets analysed during the current study are available at the PhysioBank repository, https://physionet.org/physiobank/database/chbmit/, https://doi. org/10.13026/C2K01R. PhysioBank databases are made available under the ODC Public Domain Dedication and License v1.0. [17].

\section{Consent to publish}

Not applicable.

\section{Ethics approval and consent to participate}

Not applicable.

\section{Funding}

The financial support of this paper was provided by the Future and Emerging Technologies (FET) program within the Seventh Framework Programme (FP7) for Research of the European Commission, under the FET- Proactive grant agreement TOPDRIM, number FP7-ICT-318121. Funding sources had no role in the design of this study, no role in analysis, and interpretation of data or in writing the manuscript. 


\section{Publisher's Note}

Springer Nature remains neutral with regard to jurisdictional claims in published maps and institutional affiliations.

Received: 19 April 2018 Accepted: 5 June 2018

Published online: 14 June 2018

\section{References}

1. Organisation W. H. Epilepsy key facts. http://www.who.int/en/newsroom/fact-sheets/detail/epilepsy. Accessed 29 May 2018.

2. Majumdar K, Prasad PD, Verma S. Synchronization implies seizure or seizure implies synchronization? Brain Topogr. 2014;27(1):112-22.

3. Telesford QK, Simpson SL, Burdette JH, Hayasaka S, Laurienti PJ. The brain as a complex system: using network science as a tool for understanding the brain. Brain Connectivity. 2011;1(4):295-308.

4. McSharry PE, Smith L, Tarassenko L. Comparison of predictability of epileptic seizures by a linear and a nonlinear method. IEEE Trans Bio-med Eng. 2003;50(5):628-33.

5. lasemidis LD, Sackellares JC. REVIEW : Chaos theory and epilepsy. Neurosci. 1996;2:118-26.

6. Santaniello S, Burns SP, Golby AJ, Singer JM, Anderson WS, Sarma SV. Quickest detection of drug-resistant seizures: an optimal control approach. Epilepsy Behav. 2011;22:S49-60.

7. lasemidis LD, Pardalos P, Sackellares JC, Shiau DS. Quadratic binary programming and dynamical system approach to determine the predictability of epileptic seizures. J Comb Optim. 2001;5:9-26.

8. Merelli E, Piangerelli M. Rnn-based model for self-adaptive systems-the emergence of epilepsy in the human brain. In: NCTA 2014-proceedings of the international conference on neural computation theory and applications, part of IJCCI 2014, Rome; 22-24 October 2014. p. 356-61. https:// doi.org/10.5220/0005165003560361.

9. Perea JA, Harer J. Sliding windows and persistence: an application of topological methods to signal analysis. Found Comput Math 2015;15(3):799-838

10. Rucco M, Concettoni E, Cristalli C, Ferrante A, Merelli E. Topological classification of small dc motors. In: 2015 IEEE 1st international forum on research and technologies for society and industry leveraging a better tomorrow (RTSI). IEEE; 2015. p. 192-7.

11. de Silva V, Ghrist R. Coverage in sensor networks via persistent homology. Algebraic Geom Topol. 2007;7(339-358):24.

12. Chan JM, Carlsson G, Rabadan R. Topology of viral evolution. Proc Nat Acad Sci. 2013;110(46):18566-71.

13. Ibekwe AM, Ma J, Crowley DE, Yang CH, Johnson AM, Petrossian TC, Lum PY. Topological data analysis of Escherichia coli O157: H7 and non-O157 survival in soils. Front Cell Infect Microbiol. 2014;4:122.

14. Taylor D, Klimm F, Harrington HA, Kramár M, Mischaikow K, Porter MA, Mucha PJ. Topological data analysis of contagion maps for examining spreading processes on networks. Nat Commun. 2015:6:7723.

15. Merelli E, Rucco M, Piangerelli M, Toller D. A topological approach for multivariate time series characterization: the epilepsy case study. In: Proceedings of the 9th EAl conference on bio-inspired information and communications technologies (BICT 2015). 2015.
16. TOPDRIM Website. http://www.topdrim.eu. Accessed 29 May 2018.

17. Goldberger AL, Amaral LAN, Glass L, Hausdorff JM, Ivanov PC, Mark RG, Mietus JE, Moody GB, Peng CK, Stanley HE. PhysioBank, PhysioToolkit, and PhysioNet: components of a new research resource for complex physiologic signals. Circulation. 2000;101(23):215-20. https://doi. org/10.1161/01.CIR.101.23.e215.

18. Edelsbrunner $\mathrm{H}$, Harer J. Persistent homology-a survey. Contemp Math. 2008;453:257-82

19. Carlsson G, Zomorodian A, Collins A, Guibas L. Persistence barcodes for shapes. In: Proceedings of the 2004 Eurographics/ACM SIGGRAPH symposium on geometry processing. ACM; 2004. p. 124-35.

20. Binchi J, Merelli E, Rucco M, Petri G, Vaccarino F. jholes: a tool for understanding biological complex networks via clique weight rank persistent homology. Electronic Notes Theor Comput Sci. 2014;306:5-18.

21. Rucco M, Gonzalez-Diaz R, Jimenez M-J, Atienza N, Concettoni E, Cristalli C, Ferrante A, Merelli E. A new topological entropy-based approach for measuring similarities among piecewise linear functions. Submitted. http://arxiv.org/abs/1512.07613. (2016)

22. Edelsbrunner H, Harer J. Computational topology : an introduction. Providence, R.I.: American Mathematical Society; 2010.

23. Adams H, Tausz A. Javaplex tutorial. Stanford: Stanford University; 2011.

24. Zomorodian A, Carlsson G. Computing persistent homology. Discret Comput Geom. 2005;33(2):249-74.

25. Merelli E, Rucco M, Sloot P, Tesei L. Topological characterization of complex systems: using persistent entropy. Entropy. 2015;17(10):6872-92.

26. Lockwood S, Krishnamoorthy B. Topological features in cancer gene expression data. 2014. arXiv preprint arXiv:1410.3198.

27. Rucco M, Castiglione F, Merelli E, Pettini M. Characterisation of the idiotypic immune network through persistent entropy. In: Proceedings of 11 th European conference on complex systems (ECCS 2014). Berlin: Springer; 2015. p. 117-28

28. Schmidt H, Petkov G, Richardson MP, Terry JR. Dynamics on networks: the role of local dynamics and global networks on the emergence of hypersynchronous neural activity. PLoS Comput Biol. 2014;10(11):1003947.

29. Mateo J, Sánchez-Morla E, Santos J. A new method for removal of powerline interference in ecg and eeg recordings. Comput Electrical Eng. 2015;45:235-48.

30. Keshtkaran MR, Yang Z. A fast, robust algorithm for power line interference cancellation in neural recording. J Neural Eng. 2014;11(2):026017.

31. Tausz A, Vejdemo-Johansson M, Adams H. Javaplex: a research platform for persistent homology. Book of abstracts: minisymposium on publicly available geometric/topological software, June 17th \& 19th, 2012, Chapel Hill, NC, USA. p. 7-12

32. MathWorks: Decimate documentation. http://it.mathworks.com/help/ signal/ref/decimate.html. Accessed 29 May 2018.

33. Song Y, Liò P. A new approach for epileptic seizure detection: sample entropy based feature extraction and extreme learning machine. J Biomed Sci Eng. 2010;3(06):556

34. Richman JS, Moorman JR. Physiological time-series analysis using approximate entropy and sample entropy. Am J Physiol Heart Circ Physiol. 2000;278(6):2039-49.

35. Mershon B. Vietoris-Rips complex block. http://bl.ocks.org/bmers hon/41bc67cfedf95f7d196d. Accessed 29 May 2018

Ready to submit your research? Choose BMC and benefit from

- fast, convenient online submission

- thorough peer review by experienced researchers in your field

- rapid publication on acceptance

- support for research data, including large and complex data types

- gold Open Access which fosters wider collaboration and increased citations

- maximum visibility for your research: over 100M website views per year

At BMC, research is always in progress.

Learn more biomedcentral.com/submissions 